\title{
Case report: a giant arachnoid cyst masking Alzheimer's disease
}

\author{
Anna-Sophia Wahl ${ }^{{ }^{* \dagger}}$, Martin Löffler ${ }^{2 \dagger}$, Lucrezia Hausner ${ }^{1}$, Michaela Ruttorf ${ }^{3}$, Frauke Nees ${ }^{2}$ and Lutz Frölich ${ }^{1}$
}

\begin{abstract}
Background: Intracranial arachnoid cysts are usually benign congenital findings of neuroimaging modalities, sometimes however, leading to focal neurological and psychiatric comorbidities. Whether primarily clinically silent cysts may become causally involved in cognitive decline in old age is neither well examined nor understood.

Case presentation: A 66-year old caucasian man presenting with a giant left-hemispheric frontotemporal cyst without progression of size, presented with slowly progressive cognitive decline. Neuropsychological assessment revealed an amnestic mild cognitive impairment $(\mathrm{MCl})$ without further neurological or psychiatric symptoms. The patient showed mild medio-temporal lobe atrophy on structural MRI. Diffusion tensor and functional magnetic resonance imaging depicted a rather sustained function of the strongly suppressed left hemisphere. Amyloid-PET imaging was positive for increased amyloid burden and he was homozygous for the APOE\&3-gene. A diagnosis of $\mathrm{MCl}$ due to Alzheimer's disease was given and a co-morbidity with a silent arachnoid cyst was assumed. To investigate, if a potentially reduced CSF flow due to the giant arachnoid cyst contributed to the early manifestation of $A D$, we reviewed 15 case series of subjects with frontotemporal arachnoid cysts and cognitive decline. However, no increased manifestation of neurodegenerative disorders was reported.

Conclusions: With this case report, we illustrate the necessity of a systematic work-up for neurodegenerative disorders in patients with arachnoid cysts and emerging cognitive decline. We finally propose a modus operandi for the stratification and management of patients with arachnoid cysts potentially susceptive for cognitive dysfunction.
\end{abstract}

Keywords: Arachnoid cysts, Cognitive decline, Alzheimer's disease, Functional neuro-imaging, Neural plasticity

\section{Background}

Intracranial arachnoid cysts are usually benign congenital lesions, filled with cerebrospinal fluid and covered by arachnoidal cells and collagen. They typically develop between the surface of the brain and the cranial base or on the arachnoid membrane. Widespread usage of non-invasive neuroimaging modalities have made the diagnosis of arachnoid cysts one of the most common incidental findings: While in a healthy population slightly less than $3 \%$ of subjects were found to have intracranial

\footnotetext{
* Correspondence: Anna-Sophia.Wahl@zi-mannheim.de

${ }^{\dagger}$ Anna-Sophia Wahl and Martin Löffler contributed equally to this work.

'Department of Geriatric Psychiatry, Central Institute of Mental Health,

Medical Faculty Mannheim, Heidelberg University, J5, 68159 Mannheim, Germany

Full list of author information is available at the end of the article
}

abnormalities [1], the prevalence of arachnoid cysts has been estimated to be between 0.3 and $1.7 \%$ of the general population and highest in the pediatric subpopulation [2-5]. Most intracranial arachnoid cysts remain clinically silent so that patients may live their entire life without symptoms, even if cysts are large. For symptomatic cysts, when patients present with signs of normal pressure hydrocephalus (gait disturbance, incontinence and dementia) and speech impairment, surgical cyst decompression may lead to an improvement $[6,7]$.

We here present the case of a 66-year-old man with a large left-hemispheric cyst and mild cognitive impairment. Although at first glance a cognitive decline seemed to be related to the anatomic anomaly, further extensive evaluation using different imaging modalities and biomarkers was required to reveal Alzheimer's disease

(c) The Author(s). 2019 Open Access This article is distributed under the terms of the Creative Commons Attribution 4.0 International License (http://creativecommons.org/licenses/by/4.0/), which permits unrestricted use, distribution, and reproduction in any medium, provided you give appropriate credit to the original author(s) and the source, provide a link to the Creative Commons license, and indicate if changes were made. The Creative Commons Public Domain Dedication waiver (http://creativecommons.org/publicdomain/zero/1.0/) applies to the data made available in this article, unless otherwise stated. 
rather than the arachnoid cyst as underlying cause for the cognitive deterioration. With this case report we not only aim at presenting the coincidental finding of a large cyst and a neurodegenerative disease where the neurodegenerative component might have been overlooked without adequate diagnostic tools. We also discuss that arachnoid cysts mask and may further augment neurodegenerative processes. We thus suggest a modus operandi for the diagnosis and treatment of patients with arachnoid cysts and suspected cognitive impairment.

\section{Case presentation Methods \\ Patient}

A 66-year-old man with a previously diagnosed giant arachnoid cyst presented at the memory clinic of the Department of Geriatric Psychiatry of the Central Institute for Mental Health Mannheim, University of Heidelberg, Germany, for further assessment and therapeutic counseling of mild, gradual short-term memory deficits.

\section{Clinical, EEG and laboratory assessments}

Clinical information were obtained by personal interviews and detailed medical records by trained psychiatrists. All previous clinical data were reviewed at the Central Institute of Mental Health, Mannheim. For neuropsychological assessment, the Consortium to Establish a Registry for Alzheimer's Disease (CERAD) test battery, the Mini-Mental State Examination (MMSE), the Wechsler Memory Scale logical memory (WMS-LM) and the Verbal Learning and Memory Test (VLMT), the Clock-Drawing Test and the Rey Complex Figure Test were performed. For the CERAD and WMS, raw data were transformed in specific values and converted in z-scores adapted for sex, age and education for comparison to the general population. We interviewed the patient's spouse based on the Alzheimer's Disease Cooperative Study - Activity of Daily Living (ADCSADL) checklist. The Edinburgh Handedness Inventory was used to assess the patient's dominance for handedness in everyday activities (Table 1). A routine standard EEG recording using the 10-20 system was performed and analyzed visually. A detailed laboratory screening was performed to exclude secondary dementias according to the current guideline of the German Societies of Neurology and Psychiatry (DGN/DGPPN S3-guideline revision1, http://www.awmf.org/uploads/tx_szleitlinien/ 038-0131_S3-Demenzen-2016-07.pdf).

\section{Neuroimaging}

Sensorimotor task The patient performed visually cued voluntary movements of individual digits in the scanner. The movement performed was a button press (=flex and extend back) on an optical response keypad (LUMItouch)
Table 1 Edinburgh Handedness Inventory depicting the lefthandedness of the patient. However, the patient was trained to write with his right hand at school

\begin{tabular}{|c|c|c|}
\hline & Left & Right \\
\hline Writing & & $x x$ \\
\hline Drawing & & $x x$ \\
\hline Tossing & & $x x$ \\
\hline Using scissors & $x x$ & \\
\hline Toothbrush & $x x$ & \\
\hline Knife & $x x$ & \\
\hline Spoon & & $x x$ \\
\hline Broom-leading hand & $x$ & \\
\hline To light a match & $x x$ & \\
\hline Open a box & $x$ & \\
\hline Dominant foot for kicking s.th. & & $x$ \\
\hline Usage of the dominant eye & $x$ & \\
\hline
\end{tabular}

with five response buttons, with the respective digit: digit 1 (D1: thumb), digit 2 (D2: index finger), digit 3 (D3: middle finger), digit 4 (D4: ring finger), and digit 5 (D5: little finger). Movements were performed after a visual signal. For each digit the patient received visual cues in the form of a hand drawing with the respective finger being colored. We used a block-design with movement blocks of $12 \mathrm{~s}$ and rest blocks of $10,11,12,13$ or $14 \mathrm{~s}$ (randomized) duration. The duration of the rest blocks was varied to avoid synchronization of stimulation and fMRI signals. During movement blocks, a visual cue instructed the patient to perform movements of a specific digit at $1 \mathrm{~Hz}$ (e.g., D2, D2, D2, D2.. .). Each movement block was separated by a rest block. Six movement blocks per digit were acquired (30 movement blocks in total). One block of all 5 digits was performed before any block was repeated. This was done to avoid timing effects on the BOLD signal. The order of blocks within one of these repetitions was randomized. In total the task consisted of 30 movement blocks ( 5 digits, 6 repetitions) and 31 rest blocks ( 1 in the beginning, 1 in the end of the experiment, 29 between movement blocks), summing up to a total of $12 \mathrm{~min}$ and $12 \mathrm{~s}$, which equals 488 acquired EPI volumes (see below). This task was performed twice, once with the digits of the left and once with the digits of the right hand.

\section{Magnetic resonance imaging (MRI) data acquisition}

Diagnostic MRI The magnetic resonance images for diagnosis were acquired on a $1 \mathrm{~T}$ MAGNETOM Harmony whole-body scanner (Siemens Healthineers, Erlangen, Germany) using a standard head coil. For diagnostic 
purposes, a sagittal $\mathrm{T}_{2}$ turbo spin echo (TSE) sequence (repetition time $(\mathrm{TR})=3420 \mathrm{~ms}$, echo time $(\mathrm{TE})=91 \mathrm{~ms}$, field of view $(\mathrm{FoV})=240 \times 240 \mathrm{~mm}^{2}$, flip angle $(\alpha)=170^{\circ}$, bandwidth $(B W)=150 \mathrm{~Hz} / \mathrm{px})$ was used. Nineteen slices were acquired (voxel size $=0.5 \times 0.5 \times 6.0 \mathrm{~mm}^{3}$ ). A coronal $2 \mathrm{D} \mathrm{T}_{1}$-weigthed spin echo (SE) sequence $(\mathrm{TR}=485 \mathrm{~ms}$, $\mathrm{TE}=10 \mathrm{~ms}, \quad \mathrm{FoV}=230 \times 201 \mathrm{~mm}^{2}, \alpha=90^{\circ}, \mathrm{BW}=100 \mathrm{~Hz} /$ px) was used. Twenty-three slices were acquired (voxel size $=0.5 \times 0.5 \times 6.0 \mathrm{~mm}^{3}$ ). Axial fluid attenuated inversion recovery (FLAIR) images were acquired using the following parameters: $\mathrm{TR}=8000 \mathrm{~ms}, \mathrm{TE}=124 \mathrm{~ms}$, inversion time $=$ $2500 \mathrm{~ms}, \mathrm{FoV}=230 \times 201 \mathrm{~mm}^{2}, \alpha=150^{\circ}, \mathrm{BW}=180 \mathrm{~Hz} / \mathrm{px}$. Twenty-four slices were acquired (voxel size $=0.5 \times 0.5 \times$ $6.0 \mathrm{~mm}^{3}$ ).

All diagnostic MR images were evaluated visually by an experienced neuroradiologist, medio-temporal lobe atrophy was measured by the semi-quantitative MTArating scale (Scheltens scale), the amount of white matter degeneration was rated using the semi-quantitative Fazekas scale [8].

Structural and functional MRI (fMRI) All structural and functional magnetic resonance images were acquired on a 3 T MAGNETOM Trio whole-body scanner (Siemens Healthineers, Erlangen, Germany) using a 32-channel head coil. All $\mathrm{T}_{1}$-weighted images were obtained using a highresolution magnetization prepared rapid gradient echo $(3 \mathrm{D}$ MPRAGE) sequence $(\mathrm{TR}=1900 \mathrm{~ms}, \mathrm{TE}=2.72 \mathrm{~ms}, \mathrm{FoV}=$ $250 \times 250 \mathrm{~mm}^{2}, \alpha=9^{\circ}, \quad B W=200 \mathrm{~Hz} / \mathrm{px}$ ). Two hundred and twenty-four axially-oriented slices were acquired (voxel size $=0.8 \times 0.8 \times 0.8 \mathrm{~mm}^{3}$ ). Parallel acceleration technique (iPAT) with generalized auto-calibrating partially parallel acquisition (GRAPPA) reconstruction was used with an acceleration factor of 2 . Before all measurements, shimming of the scanner was done to account for maximum magnetic field homogeneity. For acquisition of fMRI data, a gradientecho echo-planar (EPI) $\mathrm{T}_{2}{ }^{*}$ sensitive sequence $(\mathrm{TR}=1500$ $\mathrm{ms}, \mathrm{TE}=22 \mathrm{~ms}, \mathrm{FoV}=220 \times 220 \mathrm{~mm}^{2}, \alpha=90^{\circ}, \mathrm{BW}=1262$ $\mathrm{Hz} / \mathrm{px}$ ) was used. Twenty-two AC/PC aligned slices (voxel size $=1.8 \times 1.8 \times 1.8 \mathrm{~mm}^{3}$, no gap) were acquired in interleaved slice order using GRAPPA acceleration factor 3. For acquisition of diffusion weighted images, a spin-echo EPI $\left(\mathrm{TR}=8400 \mathrm{~ms}, \mathrm{TE}=84 \mathrm{~ms}, \mathrm{FoV}=192 \times 192 \mathrm{~mm}^{2}, \mathrm{BW}=\right.$ $1930 \mathrm{~Hz} / \mathrm{px}$ ) was used. Seventy slices (voxel size $=2.0 \times$ $2.0 \times 2.0 \mathrm{~mm}^{3}$, no gap) were acquired in interleaved slice order using GRAPPA acceleration factor 2. Diffusion weighting was performed in multi-directional diffusion weighting (MDDW) mode along 64 non-collinear directions with $\mathrm{b}=1000 \mathrm{~s} / \mathrm{mm}^{2}$. Additionally, a single nondiffusion weighted volume $\left(b=0 \mathrm{~s} / \mathrm{mm}^{2}\right)$ was acquired. For image registration purposes, a single-volume high-saturation EPI whole brain image was acquired with the same parameters and slice placement as in the task EPI protocol described above but with different TR (4410 ms) and containing more slices (80).

Amyloid-positron imaging tomography (A-PET) imaging A- PET scanning was performed using Florbetaben $([18 \mathrm{~F}] \mathrm{FBB})$. The amyloid imaging was acquired with a Siemens ECAT-47 PET tomograph. The [18F] FBB PET data (47 slices; slice thickness $3.3 \mathrm{~mm}$ ) was summed for $90 \mathrm{~min}$ p.i. followed by a static 3-dimensional emission-scan over $15 \mathrm{~min}$. The acquired PET data underwent standard reconstruction and images were visually assessed for amyloid positivity by a specialist trained in nuclear medicine. An established binary (amyloid-positive versus amyloid negative) scoring system was used $[9,10]$.

\section{MRI data processing and analysis}

For our analyses, we used Freesurfer software [11]. First, using the structural T1-weighted image, we generated a model of the cortical surface -involving intensity normalization, skull removal, segmentation, and tessellation. The segmentation was edited using control point procedures in freesurfer (semi-automated editing), which were followed up by manual editing.

Second, for fMRI statistical map analyses, time-series data were first motion corrected in three spatial dimensions to reduce the effects of small head motion [12]. To improve image registration the EPI images from the fMRI task were registered to the whole brain EPI image before registration to the high resolution anatomical image. Surface smoothing of $5 \mathrm{~mm}$ FWHM was applied and all the analyses were conducted within individual subject space. Activation in sub-regions was deemed significant at a threshold of $p<0.001$. Cortical parcellation was performed with an automatic labelling of the cortical structures based on the Desikan-Killiany anatomical atlas. For results of cortical parcellation for pre- and postcentral gyrus see Additional file 1: Figure S1 and as illustration of the segmentation results see Additional file 2: Figure S2 and Additional file 3: Figure S3. For DTI analyses, we used FMRIB software library 5.0.10 [13]. After eddy correction and rotation of b vectors, we used dtifit routine with weighted least-squares regression to calculate fractional anisotropy (FA) and eigenvector maps.

\section{Review of literature}

For a systematic review of literature we searched PubMed with the key words 'arachnoid cyst' and 'cognitive impairment'/'cognitive decline'/'dementia'. This revealed a total of 44 references. We excluded studies in children (9/44), papers with no detailed information of cyst location and/or symptoms of the patients $(14 / 44)$, other comorbidities (3/44) or without an English abstract available (4/44). We included 14 clinical studies and case reports (Table 3), which presented subjects with frontotemporal arachnoid 
cysts and cognitive decline or other neurological symptoms. We thus evaluated the cases of 47 patients for symptomatic presentation, diagnosis and treatment.

\section{Results}

The patient presented at the memory clinic for the assessment of slowly progressive short-term memory deficits over the course of the past 3 years. He reported troubles in finding words, forgetting names of friends and items he intended to buy in the supermarket. He described a normal developmental history with no developmental delay or psychosocial abnormalities. He remembered that he was teased by other children because of his large head and that even as a small child he would have required headgears in adult size. He denied any history of head trauma, toxic exposure, chronic headaches or another neurological or psychiatric illness. The patient had never used any psychiatric drug before. There was no history of learning disorders, hyperactivity or significant academic difficulties as well as no form of substance abuse. He completed high school and professional training as a bank accountant, where he continued to work for 27 years followed by several years of work in a communication company. The patient has stable and good social relationships. He is retired but still assisting in a pharmacy by delivering medicine to clients.

At the time of examination, his spouse confirmed cognitive decline over several years, and reported a tendency of participating less in conversations, while otherwise his psychosocial functioning was normal. She also had noticed an impaired orientation in new surroundings and episodes of confusion in group discussions or family meetings.

Three years ago he had consulted a neurologist for his memory impairment, who initiated a structural cranial MRI. This revealed a large left frontotemporal cerebrospinal fluid- filled cyst with a cross-sectional area of $6 \times 14 \mathrm{~cm}$ compatible with a Type III arachnoid cyst according to Galassi classification [14] of middle cranial fossa arachnoid cysts (Fig. 1). Two subsequent MRIs within the following 3 years did not show any size progression. On repeated neurosurgical evaluation it was decided for watchful waiting and against surgical intervention because of the absence of focal neurological signs and cyst size progression.

\section{Neurological and psychiatric examination}

Psychiatric examination was unremarkable except for mild cognitive dysfunction. At the time of evaluation, the patient's mood was reported stable and euthymic (5 out of 60 points in the Montgomery-Asberg Depression Rating Scale).

The neurological examination was normal. There were no clinical signs of hydrocephalus (no speech and gait problems, incontinence or papilledema) detected. Although the patient was mainly left-handed (Edinburgh Handedness Inventory, Table 1) he was trained to write with his right hand. However, he kept preferentially using his left hand for all fine skilled motor function and sports (tennis, squash). We also performed a detailed laboratory screening according to the current guideline of the German Societies of Neurology and Psychiatry (see methods) and could exclude hints for secondary forms of dementia.

\section{Electroencephalography (EEG) recordings}

EEG showed a distinct $10 \mathrm{~Hz}$ alpha rhythm with normal topographical distribution and no lateral or focal abnormalities and no signs of increased cerebral excitability. The visual blockade reaction was normal. There were no signs of increased slow wave activity.

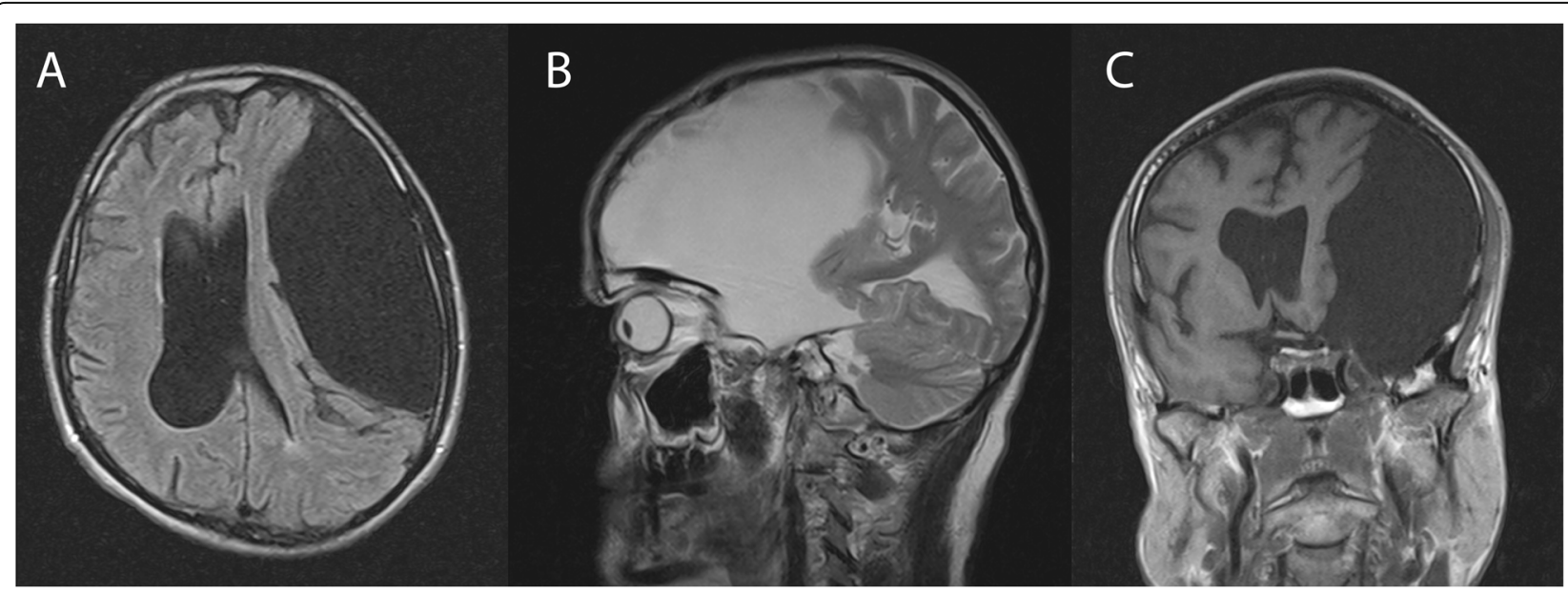

Fig. 1 Brain MRI showing a large left frontotemporal cerebrospinal fluid-filled cyst with a cross-sectional area of $6 \times 14 \mathrm{~cm}$ compatible with a Type III arachnoid cyst after the Galassi classification of middle cranial fossa arachnoid cysts: a FLAIR axial, b T2 TSE sagittal, and c T1 SE coronal view 


\section{Neuropsychological assessment}

On neuropsychological assessment no significant deficits were detectable in the CERAD test battery for most of the tests. However his performance in discriminability was mildly impaired (Table 2). The patient's overall level of cognitive function, phonetic fluency, figure savings and constructional praxis recall were on the lower border range compared to a sex-, age- and education- adapted representative sample set of the general population. With the WMS we aimed to assess the patient's mnestic performance for complex verbal learning matter. While the patient completed the immediate recall task on average level, his assessment was below average in the delayed recall after $30 \mathrm{~min}$ (<- 1 standard deviation, Table 2, B). We also tested the patient in the VLMT, which evaluates the learning performance, consolidation of longterm memory and the recognition of a list consisting of 15 words. Although the patient revealed a still adequate learning curve (Table 2, C) and an unremarkable assessment for immediate recall, his performance rate dropped meaningfully for the delayed recalling after the presentation of an interference word list, thus indicating a prominently impaired consolidation of verbal learning matter. In contrast, the patient was able to draw a complex figure in the Rey Complex Figure Test without problems in the immediate and delayed recall.

Table 2 Neuropsychological test results in the CERAD test battery

\begin{tabular}{|c|c|c|c|c|}
\hline \multicolumn{5}{|l|}{$\bar{A}$} \\
\hline & Raw & Max & z-score & Evaluation \\
\hline Verbal Fluency (animals) & 18 & - & $-0,8$ & Non-significant \\
\hline Boston Naming Test & 15 & 15 & 0,9 & Non-significant \\
\hline Mini-Mental Status & 28 & 30 & $-1,1$ & Lower border of normal range \\
\hline Wordlist test & 18 & 30 & $-0,7$ & Non-significant \\
\hline a) Wordlist recall 1st trial & 5 & 10 & $-0,2$ & Non-significant \\
\hline b) Wordlist recall 2nd trial & 6 & 10 & $-0,7$ & Non-significant \\
\hline c) Wordlist recall 3rd trial & 7 & 10 & $-0,8$ & Non-significant \\
\hline Wordlist recall & 6 & 10 & $-0,5$ & Non-significant \\
\hline Intrusions & 0 & - & 0,7 & Non-significant \\
\hline Wordlist Savngs (\%) & $86 \%$ & - & $-0,1$ & Non-significant \\
\hline Discriminability (\%) & $90 \%$ & $100 \%$ & $-1,5$ & Mild impairment \\
\hline Constructional Praxis Test & 11 & 11 & 0,7 & Non-significant \\
\hline Constructional Praxis recall & 8 & 11 & $-1,2$ & Lower border of normal range \\
\hline Figures-Savngs (\%) & $73 \%$ & - & $-1,1$ & Lower border of normal range \\
\hline Phonematic Fluency (S-Words) & 8 & - & $-1,1$ & Lower border of normal range \\
\hline Trail Making Test, Part A & 42 & 180 & $-0,1$ & Non-significant \\
\hline Trail Making Test, Part B & 72 & 300 & 1,1 & Non-significant \\
\hline Trail Making Test, B/A & 1,7 & - & 1,1 & Non-significant \\
\hline \multicolumn{5}{|l|}{ B } \\
\hline & Raw & Max & SD & Evaluation \\
\hline WMS Logical Memory Immediate recall & 21 & 50 & & avarage \\
\hline WMS Logical Memory Delayed recall & 15 & 50 & $<-1$ & Below average \\
\hline \multicolumn{5}{|l|}{ C } \\
\hline & Raw & Percentile & T-score & Evaluation \\
\hline VLMT Consecutiva learning & 38 & 20 & 41 & Lower border of normal range \\
\hline VLMT Interference & 3 & $<5$ & & Below avarage \\
\hline VLMT Recall & 5 & $<5$ & 29 & Below avarage \\
\hline \multicolumn{5}{|l|}{ D } \\
\hline & Performance & Evaluation & & \\
\hline Clock-Drawing Test & Mild vsuo-constructive deficits score $=1-2$ & average & & \\
\hline Rey Complex Figure Test & Normal range & average & & \\
\hline
\end{tabular}


In summary, the patient's test results were in particular without impairment for logic abstract cognitive functions as well as planning and implementation of actions. However, the results of the WMS and VLMT provide hints for cognitive impairment with regard to the verbal memory. In a structured interview using the ADCS-ADL scale, the patient's spouse reported declining performance in every-day life activities (achieved score $=39 / 52$ points) during the last 2 years.

\section{Structural MRI results}

As shown by two previous MRI scans, the structural MRI revealed an extended arachnoid cyst in the left frontotemporal region, with a midline shift about $12 \mathrm{~mm}$ to the right (Fig. 1). A compression of the right lateral ventricle, a displacement of the mesencephalon to the right as well as a displacement of the arteria cerebri media in the M1and M2 segments were shown. On the right cerebral hemisphere a slight global brain volume reduction was detected. The right temporal horn was slightly enlarged (Scheltens score 2) as sign of medio-temporal lobe atrophy typical for Alzheimer's disease [15]. There was no relevant microangiopathy (Fazekas score 0 ).

\section{Functional imaging}

Since the structural MRI had revealed a marked mass effect on the left hemisphere (Fig. 1) induced by the giant cyst, we next aimed at exploring impairment of functional integrity in particular to the left hemisphere due to the arachnoid cyst. Diffusion tensor imaging (DTI, Fig. 2) provided a first hint of relative well-preserved structures of fiber bundles in the left hemisphere compared to the right one.

Several studies have suggested a direct interplay between cognitive and motor functioning in the healthy elderly [16-18]. We thus performed fMRI with a sensorimotor task (visually cued movements of individual digits in the scanner, see methods) to examine functional integrity of the left hemisphere despite the giant cyst. Preserved motor representation was shown by co-localization of the motor representation of the fingers and parcellation results for primary motor and sensory cortex in the left and right hemisphere (Fig. 3), including an intact representation of the right fingers in tissue which was roughly localized at the position of the primary motor and sensory cortex (Additional file 1: Figure S1, Additional file 3: Figure S3). Although the cyst had a huge mass effect squeezing and smashing the left hemisphere towards the right one mapping of the functional areas was localized similarly in both hemispheres. Left hand movements were better represented in both hemispheres compared to the right hand, reflecting the predominant left-handedness of our patient. The bilateral presentation of both hands (although weaker in the right hand) may be explained by the relearning of right hand-usage for writing at school (Table 1). In

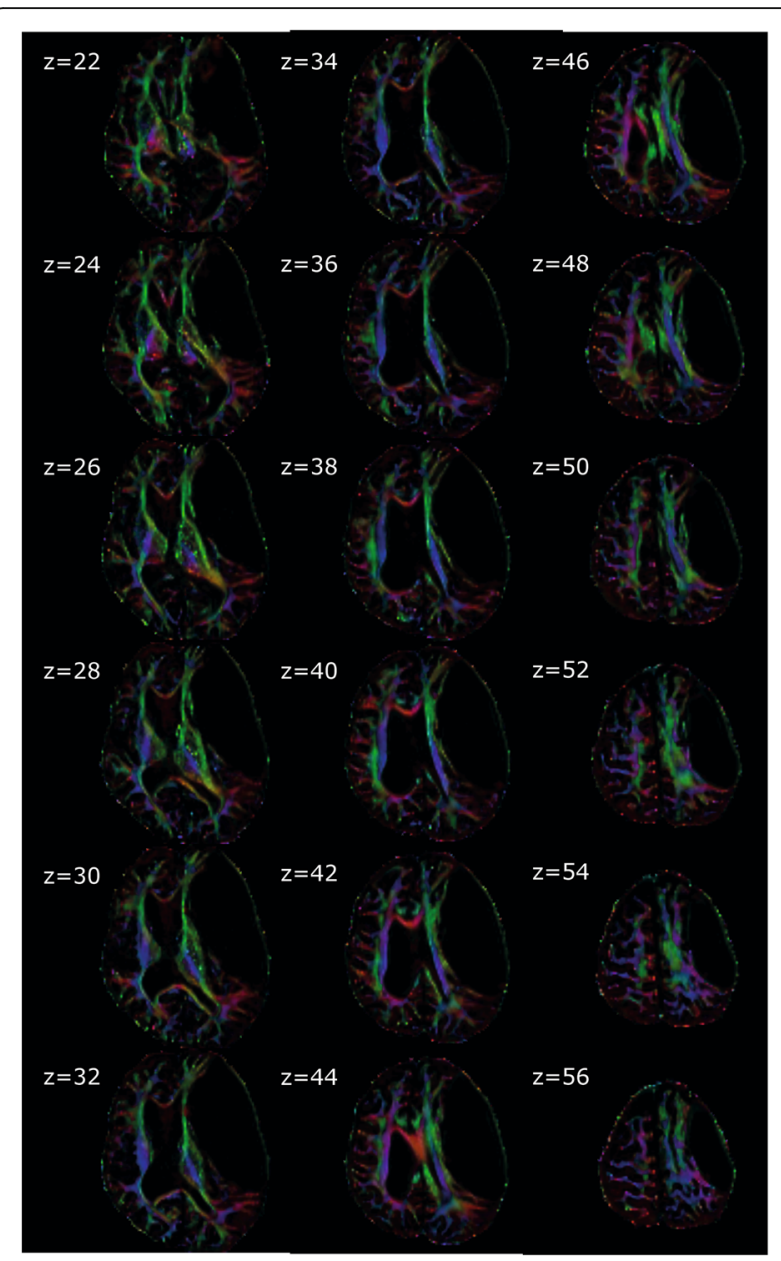

Fig. 2 Images of first eigenvector overlaid on fractional anisotropy (FA) images. The brightness of the vector colors in each voxel is modulated according to the values in the corresponding FA image, indicating the axis of diffusion. The colors red, green and blue correspond to the directions left/right, anterior/posterior and inferior/superior, respectively. The slice position in axial direction is given by its $z$ coordinate next to the image

summary, our results suggest the structural and functional preservation of the left hemisphere independently of the arachnoid cyst.

\section{Amyloid-PET result}

The results of the functional imaging made tissue damage and thus a consecutive cognitive decline due to the giant arachnoid cyst less probably. The structural magnetic resonance imaging however had elucidated a first hint for another underlying neurodegenerative cause for the beginning cognitive impairment: Medio-temporal lobe atrophy in the right hemisphere, typical for Alzheimer's disease. Because of the unfavorable risk/benefit balance ratio for lumbar puncture in this patient we decided for an amyloid-PET for investigation of Alzheimer pathophysiology. Pathologically increased 


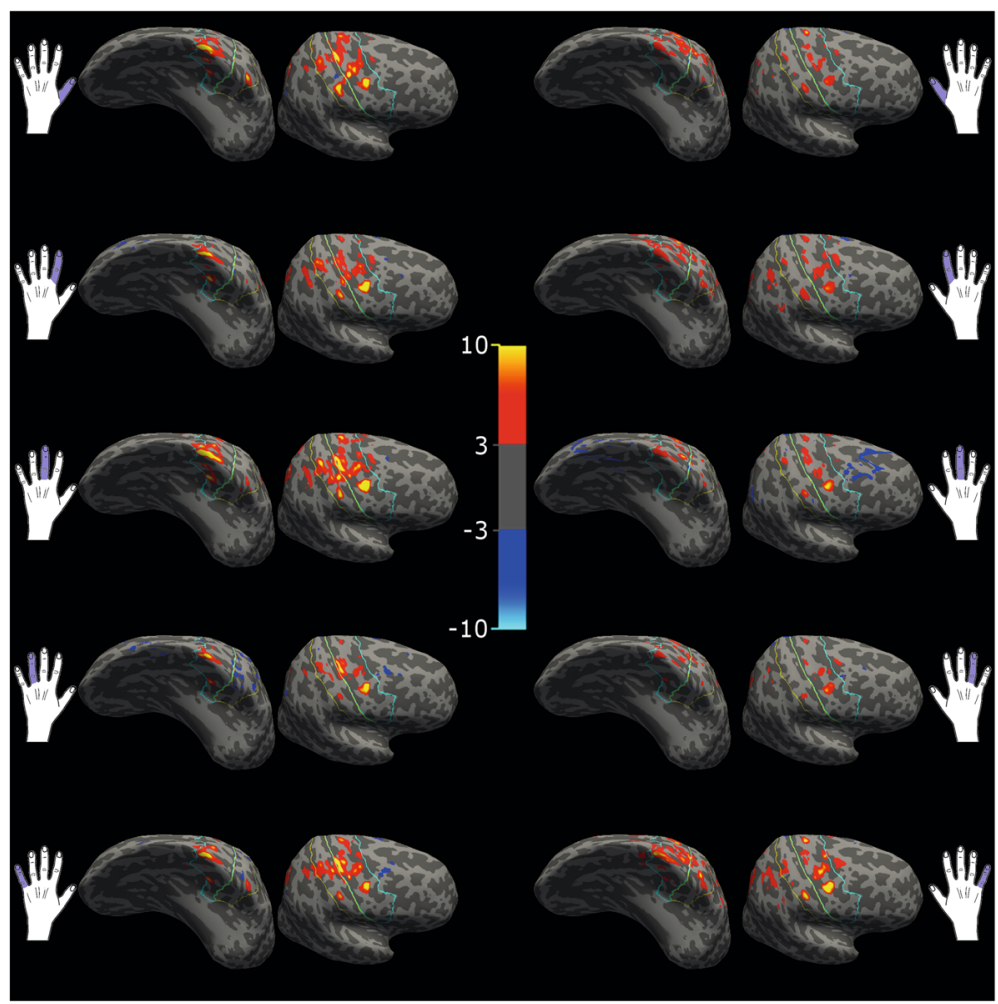

Fig. 3 Functional magnetic resonance imaging results overlaid on the inflated left and right hemisphere reconstruction. The rows 1 to 5 depict activation patterns in response to digit 1 (first row) to digit 5 (last row). The first and second columns depict activation in the left (first column) and right hemisphere (second column) in response to left hand stimulation. The third and fourth columns depict activation in the left (third column) and right hemisphere (fourth column) in response to right hand stimulation. Activation is shown as 'significances', ie, -log10(p). So, for $p=.001$, sig $=3$. Colour code ranges from 3 to 10 as illustrated by the color bar in the center. Additionally outlines of pre- and postcentral gyrus are depicted in blue and yellow, corresponding to parcellation results depicted in Additional file 1: Figure S1

cortical Amyloid deposition was detected in both white and grey matter (Fig. 4), typical for Alzheimer pathophysiology.

We also tested for an apolipoprotein E4 allele. However the patient was negative for an APoE-Genotype suggestive for an advanced manifestation of Alzheimer dementia.

The patient is now followed up in the memory clinic for further cognitive decline or progression into Alzheimer's dementia.

\section{Review of literature}

Neuroimaging has revealed a large left frontotemporal cerebrospinal fluid-filled cyst in our patient. Although arachnoid cysts may develop anywhere in the central nervous system, most cysts have been reported in the middle cranial fossa [19]. We have performed a systematic search of literature to enlighten the typical location and clinical manifestation of intracranial cysts presented in literature and potential interaction with the manifestation of dementia. We found 14 clinical studies and case reports (Table 3 [2, 6, 7, 20-29]), which presented subjects with frontotemporal arachnoid cysts and cognitive decline or other neurological symptoms. We analyzed a total of 47 patients including 28 men (59.6\%) and 19 women (40.4\%).
Average age was 50.9 years in men and 47.9 years in women. Comparable to our patient, the location of the arachnoid cysts was more pronounced in the left hemisphere (68.1\%), versus the right hemisphere (21.3\%) versus an interhemispheric location (10.6\%). Our data are in accordance with others claiming that cysts are commonly located on the left side with males most commonly having cysts in this area [25].

In the 47 patients analyzed here (Table 3 ), main predilection site was the temporal lobe $(70.2 \%)$, with the frontal lobe second $(8.5 \%)$ or in both lobes $(4.3 \%)$ versus other locations (17.0\%). $42.8 \%$ of all subjects described presented with cognitive impairment which ranged from word finding difficulties to signs of dementia. In contrast to our patient, headache was the most prominent symptom in $72.3 \%$ of patients. Other typical symptoms associated with arachnoid cysts were epilepsy and gait disturbance/vertigo (both in $14.9 \%$ of patients) as well as hemiparesis, incontinence and speech impairment (in $8.5 \%$ of the patients analyzed). However, most literature on cysts and cognitive decline is published by neuro-surgical departments. $89.3 \%$ of the patients included in the studies reviewed here received surgical treatment in form of cysto-peritoneal- 


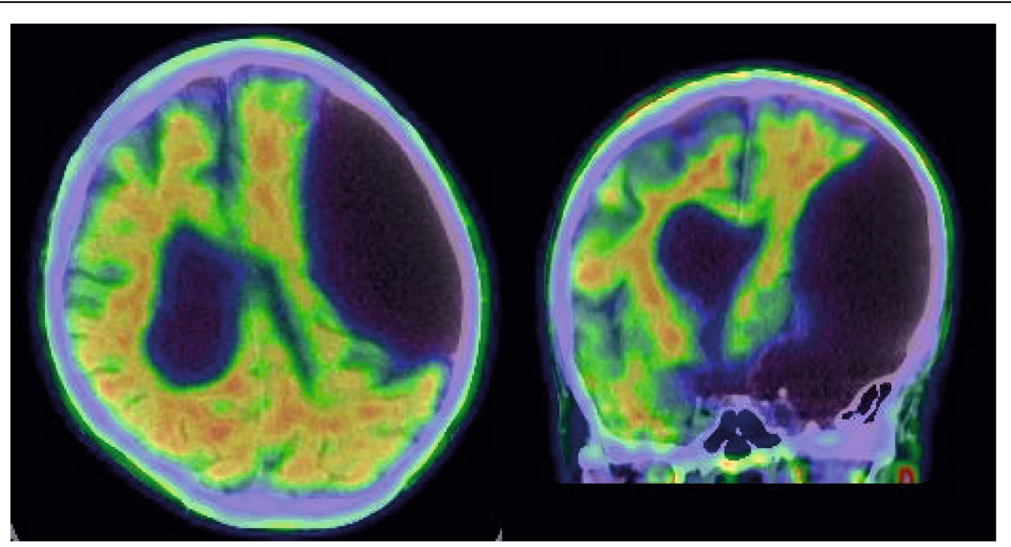

Fig. 4 Coronal and horizontal brain slices of Amyloid-PET imaging. F18-FBB (Florbetaben) tracer was used depicting amyloid-beta burden in both grey and white matter. The border between grey and white matter is indistinguishable thus indicating a pathological cortical amyloid-beta deposit

shunt surgery or cystectomy, thus indicating a reporting bias of mostly publishing cases with symptomatic cysts requiring surgical intervention. Long-term studies examining arachnoid cysts and assessing cognitive function over time are missing so far.

\section{Discussion and conclusions}

Our patient revealed two potential etiological factors for the development of cognitive decline: The giant arachnoid cyst reducing his neuronal plasticity and Alzheimer's disease [30]. As shown in our case report an extensive work-up was

Table 3 Overview of the here reviewed 15 clinical studies and case reports, which presented subjects with frontotemporal arachnoid cysts and cognitive decline as well as other neurological symptoms

\begin{tabular}{|c|c|c|c|c|c|c|c|c|}
\hline $\begin{array}{l}\text { Piece of } \\
\text { literature }\end{array}$ & $\begin{array}{l}\text { \# of } \\
\text { patients }\end{array}$ & $\begin{array}{l}\text { Age of } \\
\text { patients }\end{array}$ & Sex & Hemisphere & Interhemispheric & $\begin{array}{l}\text { Cognitive } \\
\text { impairment }\end{array}$ & Other symptoms & Surgery \\
\hline Amin, 2013 & 1 & 65 & $\mathrm{~m}$ & right & no & yes & $\begin{array}{l}\text { spastic left sided hemiparesis, late } \\
\text { onset seizures }\end{array}$ & no \\
\hline $\begin{array}{l}\text { Bohnen, } \\
2016\end{array}$ & 1 & 70 & $\mathrm{~m}$ & left & no & yes & gait disturbance & no \\
\hline Gjerde, 2013 & 22 & $22-68$ & $\begin{array}{l}12 \times \mathrm{m} / \\
10 \times f\end{array}$ & $\begin{array}{l}14 x \text { left, } 8 x \\
\text { right }\end{array}$ & no & yes (4 pat.) & $\begin{array}{l}\text { vertigo ( } 2 \text { pat.), headache ( } 19 \text { pat.), } \\
\text { epilepsy ( } 2 \text { pat.) }\end{array}$ & yes \\
\hline Golaz, 1993 & 1 & & f & & yes & yes & no & no \\
\hline $\begin{array}{l}\text { Hishikawa, } \\
2002\end{array}$ & 1 & 64 & f & left & no & yes & headache, speech disturbance & yes \\
\hline $\begin{array}{l}\text { Hishikawa, } \\
2002\end{array}$ & 1 & 73 & f & & yes & no & right hemiparesis & yes \\
\hline Kotil, 2007 & 1 & 70 & f & & yes & yes & vertigo, behavioral disturbance & yes \\
\hline $\begin{array}{l}\text { Lebowitz, } \\
2006\end{array}$ & 1 & 33 & $\mathrm{~m}$ & left & no & yes & no & no \\
\hline Miyaji, 2012 & 1 & 68 & $\mathrm{~m}$ & left & no & yes & $\begin{array}{l}\text { headache, acalculia, agraphia, finger } \\
\text { agnosia }\end{array}$ & no \\
\hline $\begin{array}{l}\text { Ramtahal, } \\
2006\end{array}$ & 1 & 61 & f & & yes & yes & gait disturbance, incontinence & yes \\
\hline $\begin{array}{l}\text { Shirakawa, } \\
1991\end{array}$ & 1 & 72 & f & right & no & yes & gait disturbance, incontinence & yes \\
\hline $\begin{array}{l}\text { Sugimoto, } \\
2016\end{array}$ & 1 & 72 & $\mathrm{~m}$ & & yes & yes & gait disturbance, dizziness & yes \\
\hline Wester, 1995 & 13 & & $\begin{array}{l}11 \times m / \\
2 \times f\end{array}$ & left & no & no & seizures (3 pat.), hemiparesis (2 pat.), & yes \\
\hline $\begin{array}{l}\text { Zwagerman, } \\
2016\end{array}$ & 1 & 49 & f & left & no & no & $\begin{array}{l}\text { staring spells, expressive dysphasia, } \\
\text { tremor }\end{array}$ & yes \\
\hline
\end{tabular}


required to dissect the contribution of each disease entity although their interaction remains a matter of debate. Our case particularly illustrates that a giant arachnoid cyst may leave the brain tissue intact but might mask an underlying neurodegenerative disorder such as Alzheimer's dementia. It also illustrates the diagnostic value of biomarkers, e.g. medio-temporal lobe atrophy (Scheltens Score 2) and increased Amyloid-PET imaging. Thus, the diagnosis of prodromal Alzheimer dementia (AD) / mild cognitive impairment due to $\mathrm{AD}$ was established in this patient [31].

Although elevated tracer binding can also be found in normal older volunteers without cognitive deficits, with the proportion of "positive cases" ranging from 10 to $30 \%$ depending on the age of the cohort and the threshold for defining the pathological tracer enhancement [32-35], the preponderance of current literature proposes that enhanced amyloid-beta signal in non-demented subjects is associated with decreased episodic memory performance and structural/functional brain changes suggestive of incipient Alzheimer's disease [36]: In a retrospective study [37], amyloid-beta positive PET scans were more common in subjects with declining cognitive test scores $(70 \%)$ than in those with stable scores (17\%).

Our findings in the cognitive assessment are in line with previous studies showing a rather heterogeneous picture of cognitive deficits across different domains, lacking clear specificity of cognitive and neuropsychological changes following prodromal $\mathrm{AD} / \mathrm{MCI}$ due to AD. The often expansive lesions due to arachnoid cysts may cause a reorganization of cortical functions. This reorganization can vary to a large extent between patients, which is reflected in changes in a variety of cognitive functions. According to a review article [38], these functions encompass verbal perception and memory, complex verbal tasks, visuospatial functions and visual attention and dichotic perception and memory [7] of which not all have to be impaired in every patient. The interpretation of cognitive impairments thus provide some evidence on the specificity of the reorganization and compensation, yet, this needs to be treated with caution as various factors co-determine such effects.

Furthermore, the application of different imaging modalities and biomarkers helped the neurosurgeons to triage our patient: the patient was followed-up by the neurosurgeons several times for a surgical intervention as clinically a mild cognitive impairment became apparent. However, when Alzheimer's disease was revealed as the leading underlying cause of cognitive decline rather than tissue damage from the arachnoid cyst, the neurosurgeons decided against a cystectomy. As there was no cyst size progression and no further neurological symptoms a benefit from the surgery was highly questionable for the patient.
Moreover, this case study is also an impressive demonstration of neuronal plasticity fully compensating for even large intracranial pathologies. Most intracranial arachnoid cysts remain clinically silent [1], reflecting the tremendous plastic capacity of the central nervous system to compensate for even large structural abnormalities. As arachnoid cysts are usually congenital, the brain fully adapts to the special circumstances during development, a condition which could be also found in our patient: The 66-year old lived his life rather unaffected by the giant arachnoid cyst, he would not be aware of until the age of 63. The patient remembers of wearing headdresses of adults at a very young age and being teased because of his head size as a child suggesting the congenital nature of the arachnoid cyst. Similar phenomena have been described in children with intractable seizures who underwent surgery with resections of entire brain regions or callosotomy $[39,40]$.

The application of different neuroimaging modalities revealed structural and functional perseveration of the left hemisphere despite being displaced and squeezed by the giant cyst: Representation of finger movements was sustained at a rather similar position in the left hemisphere compared to the right sensorimotor cortex. Interestingly, bilateral representation in particular of the left hand was revealed and even pronounced in the right hemisphere, which might be explained by the ambidexterity of the patient, being originally left-handed but trained righthanded. We chose a sensorimotor task for the fMRI experiment to map in particular the left hemisphere, as the giant arachnoid cyst had a strong mass effect on this brain region. The goal of the fMRI was to detect any functional map shift and loss of functional integrity (together with structural alterations found in the DTI (Fig. 2)), which could also impact cognitive functions. Since we did not aim at detecting the neuronal correlate of the cognitive decline but at understanding the structural and functional integrity despite the giant cyst, a cognitive task with fMRI would have been less helpful. Although we cannot rule out that memory function is still impaired despite of the intact motor function, normal sensorimotor integration may be also correlative for cognitive performance as discussed by others $[16,18]$ and vice versa: Abnormal sensorimotor integration correlates with the cognitive profile in neurological diseases [41].

However, it remains a matter of debate, if the plastic compensational mechanisms of the brain decline over time, and thus expose patients with even clinically silent brain pathologies to cognitive impairment. Although a connection between arachnoid cysts and cognitive sequelae has been proposed for over 20 years [42], the impact of clinically silent cysts on cognitive decline remains largely unknown due to the subtlety of dysfunction. Rabiei et al. [43] found that subjects with and without cysts had the 
same frequency of headache, dizziness, previous head trauma, cognitive impairment and depressive symptoms without an increased prevalence of dementia, depression, epilepsy or previous hip fracture. Nonetheless a relationship between the onset of Alzheimer's pathology and the arachnoid cyst might be plausible: As previously postulated by Silverberg et al., 2003 [44] we speculate that the existence of the arachnoid cyst may modify intracranial pressure ratios, leading to reduced perfusion and metabolism in the surrounding cortical regions. Furthermore, the cyst might perturb CSF circulatory pathology which might result - along with genetic factors such as apolipoprotein E4 A $\beta$-in the accumulation of amyloid- $\beta$ peptide, microtubular associated protein $\mathrm{\tau}$ (MAP $\mathrm{\tau}$ ) and other toxins. An increase in the steady state concentration of $A \beta$ influences $A \beta$ aggregation which is causally und pathognomonic in Alzheimer's disease $[45,46]$. In particular soluble $A \beta$ is potentially neurotoxic affecting normal neuronal function and even causing cell death $[47,48]$. Since our patient presented with apolipoprotein E $\varepsilon 3 / 3$ wildtype allele, this genotype will not have contributed to the early manifestation of Alzheimer dementia. Thus it may be speculated that the impaired CSF flow dynamics due to the giant cyst might have caused reduced amyloid clearance inducing Alzheimer pathology. Longitudinal studies in patients with huge arachnoid cysts might become important to detect symptoms of cognitive decline early and thereby open a window of opportunity for early therapeutic intervention to prevent or slow down further cognitive deterioration [49].

One major lesson to be learned from the case illustrated here is, that patients presenting with large intracranial brain cysts and cognitive decline require a closer look and pure clinical examination and structural MRI might be insufficient to triage patients either to neurosurgery or watchful waiting. The review and study presented here may thus initiate further discussion for a modus operandi for patients with large intracranial cysts: We suggest [1] a detailed neurological and neuropsychological examination as well as MRI of the brain [2]. The repetition of all procedures is crucial to detect the development of cognitive decline and neurological symptoms over time. The cyst may also become symptomatic due to growth progression and may thus require surgical intervention [3]. An additional neurodegenerative component should be evaluated either by biomarkers in the cerebrospinal fluid or amyloid-PET imaging [4]. Additional fMRI may become a useful assessment to study functional integrity and for risk analysis of cognitive deterioration: Patients with less prominent functional map changes and no neurodegenerative comorbidity may be at lower risk for a cognitive decline. In contrast, large functional map shifts may be associated with cognitive impairment due to a decline of plastic compensation in aging.
The case presented here illustrates that in patients with large intracranial brain cysts and cognitive impairment pure clinical examination and structural MRI might be insufficient to triage patients. Our case report suggests the diagnostic value of rigorous neuroimaging modalities including DTI, fMRI and amyloid-PET imaging as well as biomarkers in the cerebrospinal fluid to distinguish tissue damage induced by large cysts versus a neurodegenerative pathology as underlying cause of cognitive decline.

\section{Additional files}

\begin{abstract}
Additional file 1: Figure S1. Images of parcellation results for precentral and postcentral gyrus overlaid on T1 weighted structural image. The colors blue and yellow correspond to the pre- and postcentral gyrus. The slice position in axial direction is given by its $z$ coordinate next to the image. (PDF $10040 \mathrm{~kb}$ )
\end{abstract}

Additional file 2: Figure S2. Images of segmentation results. The colors red, white and purple correspond to the grey matter, white matter and cerebrospinal fluid. Additionally parcellation results of precentral (cyan) and postcentral (yellow) gyrus are depicted. Slice position is given by $x, y$ and z coordinates. (PDF $221 \mathrm{~kb}$ )

Additional file 3: Figure S3. Images of atlas mapping in volume space and surface space. The colors correspond to the different areas as assigned by atlas mapping. The top left shows respective results in surface space of the left hemisphere, the top right shows respective results in surface space of the right hemisphere. The bottom shows an exemplary axial view of atlas mapping in volume space at $z=70$. Red and blue areas depict precentral (blue) and postcentral (red) gyrus. (PDF 2914 kb)

\section{Abbreviations}

AD: Alzheimer Dementia; ADL: Activity of Daily Living; A-PET: AmyloidPositron Imaging Tomography; BW: Bandwidth; CERAD: Consortium to Establish a Registry for Alzheimer's Disease; DTI: Diffusion tensor imaging; FoV: Field of view; MCl: Mild cognitive impairment; MDDW: Multi-directional diffusion weighting; MMSE: Mini-Mental State Examination; SE: Spin echo; TE: Echo time; TR: Repetition time; TSE: Turbo spin echo sequence; VLMT: Verbal Learning and Memory Test; WMS-LM: Wechsler Memory Scale logical memory

\section{Acknowledgements}

Not applicable.

\section{Authors' contributions}

A.S.W. performed literature research, analyzed clinical data and wrote the manuscript. M.L. acquired and analyzed functional neuroimaging data. L.H. helped to discuss and record clinical data, F.N. and M.R. helped analyzing and interpreting data with an emphasis on neuroimaging. L.F. advised and discussed data. All authors have read and approved the manuscript.

\section{Funding}

A.S.W. is a Branco Weiss fellow and a recipient of the Margarete-vonWrangell scholarship which provides part of her employment to pursue scientific questions- as in this case the design of study, collection of data, analysis and writing of the manuscript.

\section{Ethics approval and consent to participate}

Informed consent was obtained from the subject and his wife to present his medical case according to the statues of the ethics commission of the Faculty of Behavioral and Cultural studies at the University of Heidelberg. Written consent to participate was obtained from the subject. 


\section{Consent for publication}

Written consent to publication was obtained from the subject and his wife. Furthermore, the patient's capacity for consent had been confirmed by an overseeing psychiatrist. Availability of data and material All data generated or analysed during this study are included in this published article and its Additional files.

\section{Competing interests}

The authors have no patents pending or financial conflicts to disclose. This research did not receive any specific grant from funding agencies in the public, commercial, or not-for-profit sectors.

\section{Author details}

${ }^{1}$ Department of Geriatric Psychiatry, Central Institute of Mental Health, Medical Faculty Mannheim, Heidelberg University, J5, 68159 Mannheim, Germany. ${ }^{2}$ Department of Cognitive and Clinical Neuroscience, Central Institute of Mental Health, Medical Faculty Mannheim, Heidelberg University, Mannheim, Germany. ${ }^{3}$ Computer Assisted Clinical Medicine, Medical Faculty Mannheim, Heidelberg University, Mannheim, Germany.

\section{Received: 15 August 2018 Accepted: 19 August 2019} Published online: 05 September 2019

\section{References}

1. Weber $\mathrm{F}, \mathrm{Knopf} \mathrm{H}$. Incidental findings in magnetic resonance imaging of the brains of healthy young men. J Neurol Sci. 2006;240(1-2):81-4.

2. Zwagerman NT, Pardini J, Mousavi SH, Friedlander RM. A refractory arachnoid cyst presenting with tremor, expressive dysphasia, and cognitive decline. Surg Neurol Int. 2016;7(Suppl 15):S431-3.

3. Al-Holou WN, Yew AY, Boomsaad ZE, Garton HJ, Muraszko KM, Maher CO. Prevalence and natural history of arachnoid cysts in children. J Neurosurg Pediatr. 2010:5(6):578-85

4. Katzman GL, Dagher AP, Patronas NJ. Incidental findings on brain magnetic resonance imaging from 1000 asymptomatic volunteers. JAMA : the journal of the American Medical Association. 1999;282(1):36-9.

5. Vernooij MW, Ikram MA, Tanghe HL, Vincent AJ, Hofman A, Krestin GP, et al. Incidental findings on brain MRI in the general population. N Engl J Med. 2007;357(18):1821-8.

6. BG P, Schmid M, Hammar A, Wester K. Intracranial arachnoid cysts: impairment of higher cognitive functions and postoperative improvement. J Neurodev Disord. 2013;5(1):21.

7. Wester K, Hugdahl K. Arachnoid cysts of the left temporal fossa: impaired preoperative cognition and postoperative improvement. J Neurol Neurosurg Psychiatry. 1995;59(3):293-8.

8. Fazekas F, Kleinert R, Offenbacher $H$, Schmidt R, Kleinert G, Payer F et al. Pathologic correlates of incidental MRI white matter signal hyperintensities. Neurology. 1993;43(9):1683-9.

9. Barthel H, Luthardt J, Becker G, Patt M, Hammerstein E, Hartwig K, et al. Individualized quantification of brain beta-amyloid burden: results of a proof of mechanism phase 0 florbetaben PET trial in patients with Alzheimer's disease and healthy controls. Eur J Nucl Med Mol Imaging. 2011;38(9):1702-14.

10. Barthel H, Gertz HJ, Dresel S, Peters O, Bartenstein P, Buerger K, et al. Cerebral amyloid-beta PET with florbetaben (18F) in patients with Alzheimer's disease and healthy controls: a multicentre phase 2 diagnostic study. Lancet Neurol. 2011;10(5):424-35.

11. Dale AM, Fischl B, Sereno MI. Cortical surface-based analysis. I. Segmentation and surface reconstruction. Neurolmage. 1999:9(2):179-94.

12. Cox RW, Jesmanowicz A. Real-time $3 D$ image registration for functional MRI. Magn Reson Med : official journal of the Society of Magnetic Resonance in Medicine / Society of Magnetic Resonance in Medicine. 1999;42(6):1014-8.

13. Jenkinson M, Beckmann CF, Behrens TE, Woolrich MW, Smith SM. Fsl. Neuroimage. 2012;62(2):782-90.

14. Galassi E, Tognetti F, Gaist G, Fagioli L, Frank F, Frank G. CT scan and metrizamide CT cisternography in arachnoid cysts of the middle cranial fossa: classification and pathophysiological aspects. Surg Neurol. 1982;17(5):363-9.

15. Claus JJ, Staekenborg SS, Holl DC, Roorda JJ, Schuur J, Koster P, et al. Practical use of visual medial temporal lobe atrophy cut-off scores in
Alzheimer's disease: validation in a large memory clinic population. Eur Radiol. 2017:27(8):3147-55.

16. Schaefer $S$, Schumacher $V$. The interplay between cognitive and motor functioning in healthy older adults: findings from dual-task studies and suggestions for intervention. Gerontology. 2011;57(3):239-46.

17. Buckley RF, Schultz AP, Hedden T, Papp KV, Hanseeuw BJ, Marshall G, et al. Functional network integrity presages cognitive decline in preclinical Alzheimer disease. Neurology. 2017;89(1):29-37.

18. Shah T, Verdile G, Sohrabi H, Campbell A, Putland E, Cheetham C, et al. A combination of physical activity and computerized brain training improves verbal memory and increases cerebral glucose metabolism in the elderly. Transl Psychiatry. 2014;4:e487.

19. Passero S, Filosomi G, Cioni R, Venturi C, Volpini B. Arachnoid cysts of the middle cranial fossa: a clinical, radiological and follow-up study. Acta Neurol Scand. 1990:82(2):94-100.

20. Amin OSM. Late onset seizures and progressive cognitive decline: is it an arachnoid cyst? Case Rep. 2013;2013. https://doi.org/10.1136/bcr-2013-009847.

21. Bohnen $\mathrm{Nl}$, Haugen J, Kluin K, Kotagal V. Motor speech apraxia in a 70-year-old man with left dorsolateral frontal arachnoid cyst: a [(18) F] FDG PET-CT study. Case Rep Neurol Med. 2016;2016:8941035.

22. Golaz J, Bouras C. Frontal arachnoid cyst. A case of bilateral frontal arachnoid cyst without clinical signs. Clin Neuropathol. 1993;12(2):73-8.

23. Hishikawa T, Chikama M, Tsuboi M, Yabuno N. Two cases of symptomatic arachnoid cysts in elderly patients--a comparison and analysis with child cases. No Shinkei Geka. 2002;30(9):959-65.

24. Kotil K, Balci N, Bilge T. Intracranial symptomatic giant arachnoid cyst of the interhemispheric fissure presenting with frontal lobe syndrome. Turk Neurosurg. 2007;17(2):147-51.

25. Lebowitz BK, Schefft BK, Testa SM, Patton JA, Yeh HS. Neurocognitive sequelae of a giant arachnoid cyst: case study. Neurocase. 2006;12(6):339-45.

26. Miyaji Y, Miyasaki H, Ning Z, Watanabe D, Suzuki Y, Kuroiwa Y. A 68 year-old man presenting ideomotor apraxia and incomplete Gerstmann syndrome with multiple cystic lesions in the left hemisphere. Rinsho Shinkeigaku. 2012;52(9): $681-4$.

27. Ramtahal J. Arachnoid cyst mimicking normal pressure hydrocephalus. A case report and review of the literature. J Neurosurg Sci. 2006;50(3):79-81 discussion.

28. Shirakawa N, Mukai K, Fujisawa H, Furuichi S. A case of intraventricular cyst associated with normal pressure hydrocephalic condition. No Shinkei Geka. 1991;19(9):897-902.

29. Sugimoto T, Uranishi R, Yamada T. Gradually Progressive Symptoms of Normal Pressure Hydrocephalus Caused by an Arachnoid Cyst in the Fourth Ventricle: a Case Report. World Neurosurg. 2016;85(364):e19-22.

30. Jacquemont T, De Vico FF, Bertrand A, Epelbaum S, Routier A, Dubois B, et al. Amyloidosis and neurodegeneration result in distinct structural connectivity patterns in mild cognitive impairment. Neurobiol Aging. 2017;55:177-89.

31. Dubois $B$, Hampel $H$, Feldman $H H$, Scheltens $P$, Aisen $P$, Andrieu $S$, et al. Preclinical Alzheimer's disease: definition, natural history, and diagnostic criteria. Alzheimers Dement. 2016:12(3):292-323.

32. Pike $\mathrm{KE}$, Savage $\mathrm{G}$, Villemagne $\mathrm{VL}, \mathrm{Ng} \mathrm{S}$, Moss $\mathrm{SA}$, Maruff $P$, et al. Betaamyloid imaging and memory in non-demented individuals: evidence for preclinical Alzheimer's disease. Brain : a journal of neurology. 2007;130(Pt 11):2837-44.

33. Hulette CM, Welsh-Bohmer KA, Murray MG, Saunders AM, Mash DC, McIntyre LM. Neuropathological and neuropsychological changes in "normal" aging: evidence for preclinical Alzheimer disease in cognitively normal individuals. J Neuropathol Exp Neurol. 1998;57(12):1168-74.

34. Rowe CC, Ackerman U, Browne W, Mulligan R, Pike KL, O'Keefe G, et al. Imaging of amyloid beta in Alzheimer's disease with 18F-BAY94-9172, a novel PET tracer: proof of mechanism. The Lancet Neurology. 2008;7(2):129-35.

35. Aizenstein HJ, Nebes RD, Saxton JA, Price JC, Mathis CA, Tsopelas ND, et al. Frequent amyloid deposition without significant cognitive impairment among the elderly. Arch Neurol. 2008;65(11):1509-17.

36. Rabinovici GD, Jagust WJ. Amyloid imaging in aging and dementia: testing the amyloid hypothesis in vivo. Behav Neurol. 2009;21(1):117-28.

37. Villemagne $V L$, Pike KE, Darby D, Maruff $P$, Savage $G$, Ng S, et al. Abeta deposits in older non-demented individuals with cognitive decline are indicative of preclinical Alzheimer's disease. Neuropsychologia. 2008; 46(6):1688-97.

38. Wester K. Intracranial arachnoid cysts--do they impair mental functions? J Neurol. 2008;255(8):1113-20. 
39. Graham D, Tisdall MM, Gill D. Corpus callosotomy outcomes in pediatric patients: a systematic review. Epilepsia. 2016;57(7):1053-68.

40. Griffin S, Tranel D. Age of seizure onset, functional reorganization, and neuropsychological outcome in temporal lobectomy. J Clin Exp Neuropsychol. 2007;29(1):13-24.

41. Benitez-Rivero S, Palomar FJ, Martin-Rodriguez JF, Alvarez de Toledo P, Lama MJ, Huertas-Fernandez I, et al. Abnormal sensorimotor integration correlates with cognitive profile in vascular parkinsonism. J Neurol Sci. 2017; 377:161-6.

42. Lang W, Lang M, Kornhuber A, Gallwitz A, Kriebel J. Neuropsychological and neuroendocrinological disturbances associated with extracerebral cysts of the anterior and middle cranial fossa. Eur Arch Psychiatry Neurol Sci. 1985; 235(1):38-41.

43. Rabiei K, Jaraj D, Marlow T, Jensen C, Skoog I, Wikkelso C. Prevalence and symptoms of intracranial arachnoid cysts: a population-based study. J Neurol. 2016;263(4):689-94.

44. Silverberg GD, Mayo M, Saul T, Rubenstein E, McGuire D. Alzheimer's disease, normal-pressure hydrocephalus, and senescent changes in CSF circulatory physiology: a hypothesis. The Lancet Neurology. 2003;2(8):506-11.

45. Selkoe DJ. Toward a comprehensive theory for Alzheimer's disease. Hypothesis: Alzheimer's disease is caused by the cerebral accumulation and cytotoxicity of amyloid beta-protein. Ann N Y Acad Sci. 2000;924:17-25.

46. Patterson BW, Elbert DL, Mawuenyega KG, Kasten T, Ovod V, Ma S, et al. Age and amyloid effects on human central nervous system amyloid-beta kinetics. Ann Neurol. 2015;78(3):439-53.

47. Lambert MP, Barlow AK, Chromy BA, Edwards C, Freed R, Liosatos M, et al. Diffusible, nonfibrillar ligands derived from Abeta1-42 are potent central nervous system neurotoxins. Proc Natl Acad Sci U S A. 1998;95(11):6448-53.

48. Walsh DM, Klyubin I, Fadeeva JV, Cullen WK, Anwyl R, Wolfe MS, et al. Naturally secreted oligomers of amyloid beta protein potently inhibit hippocampal long-term potentiation in vivo. Nature. 2002;416(6880):535-9.

49. Epelbaum S, Genthon R, Cavedo E, Habert MO, Lamari F, Gagliardi G, et al. Preclinical Alzheimer's disease: a systematic review of the cohorts underlying the concept. Alzheimers Dement. 2017;13(4):454-67.

\section{Publisher's Note}

Springer Nature remains neutral with regard to jurisdictional claims in published maps and institutional affiliations.

Ready to submit your research? Choose BMC and benefit from:

- fast, convenient online submission

- thorough peer review by experienced researchers in your field

- rapid publication on acceptance

- support for research data, including large and complex data types

- gold Open Access which fosters wider collaboration and increased citations

- maximum visibility for your research: over $100 \mathrm{M}$ website views per year

At $\mathrm{BMC}$, research is always in progress.

Learn more biomedcentral.com/submissions 